interviews used questions and probes on vaccine knowledge, acceptability, social and community concerns.

Results Fifteen in-depth interviews (15 MSM) were conducted. In general, there was confusion among the majority of participants whether HPV vaccine cures, treats, or prevents. Some participants had previously heard about the HPV vaccine but the majority had not known about it. Reasons mentioned for accepting the vaccine were the prevention of genital warts and related cancers; and avoiding infecting others with HPV. Vaccine cost was mentioned as a major factor related to vaccine uptake. Several participants thought receiving the vaccine would encourage condom use to avoid other STDs including HIV. Other participants predicted increased sexual risk-taking due to a bolstered sense of safety. None of the participants mentioned any stigma or social issue with this vaccine.

Conclusion The chief finding is that the acceptability of a preventive HPV vaccine was widespread but not universal among these populations depending on a range of factors. Although HPV vaccination was incorrectly perceived as therapeutic, reasons for wanting HPV vaccination centered on self-protection and the protection of sexual partners. Chief among the barriers to HPV vaccination was the cost of the vaccine rather than the vaccine-induced reactions as has been mentioned in previous studies. Thus, HPV vaccination for MSM will be out of reach except for the few who can pay for it unless either all adolescent boys (regardless of sexual and gender orientation) are included.

Disclosure No significant relationships.

\section{P254 BARRIERS TO SEXUAL ASSAULT DISCLOSURE WITHIN SEXUAL HEALTH SERVICES: A MIXED METHOD/ POPULATION STUDY}

${ }^{1}$ Jane Meyrick*, ${ }^{2}$ Kieran Mccartan, ${ }^{1}$ Zoe Thomas, ${ }^{2}$ Aga Kowalska. ${ }^{1}$ University of the West of England, Psychology, Bristol, UK; ${ }^{2}$ University of the West of England, Health and Social Sciences, Bristol, UK

\subsection{6/sextrans-2019-sti.387}

Background Internationally, the UN reports that an estimated 1 in 3 women experience physical/sexual violence during their lifetime. These rates vary across cultures, age, gender and sexual identity. However, what does not vary is that the majority (UK, 83\%) will not report this. It is likely that many will attend mainstream sexual health services for crisis STI screening or emergency contraception. It is clear that a range of psychological and health impacts may be suffered by this group including triple the risk of depression and half survivors not attending cervical screening programmes. Getting the correct help earlier is likely to reduce psychological/physical harm but there is no little or no research around victims interaction with both specialist but importantly mainstream sexual health services.

Methods A population in which sexual assault is high (students) were anonymously surveyed to establish rates of sexual assault/harassment. Participants were asked about reporting behaviour in relation to attendance at SH services. Follow up qualitative interviews examined barriers to services. Qualitative data from a parallel study of actual and potential users of a specialist sexual assault service (Sexual Assault Referral Centre) in which attempts were made to recruit from multiply vulnerable populations at high risk of sexual violence (through support projects around homelessness, drug use, sex workers etc.) were examined for information around barriers to services and disclosure rates.

Results The data around rates of self-reporting victim/survivors attending mainstream sexual health services but not disclosing that assault will be presented and examined for patterns. Analysis of qualitative interview data will be presented in order to establish why rates are low and what might improve reporting.

Conclusion What does a mainstream SH service need to do to in order to show victims of sexual assault that disclosure will be supported and further help is available.

Disclosure No significant relationships.

\section{P257 PATTERNS AND CORRELATES OF GENDER-BASED VIOLENCE (GBV) IN RURAL AND URBAN SOUTH AFRICAN COMMUNITIES}

${ }^{1}$ Saheed Usman*, ${ }^{1}$ Ndumiso Tshuma, ${ }^{1}$ Peter Nubi, ${ }^{1}$ Jessica Yun, ${ }^{2}$ Zachariah Sekhu, ${ }^{3}$ Lesiba Masibe. 'Best Health Solutions, Johannesburg, South Africa; ${ }^{2}$ Waterberg Welfare Society, Vaalwater, South Africa; ${ }^{3}$ School of Public Health University of the Witwatersrand, Johannesburg, South Africa

\subsection{6/sextrans-2019-sti.388}

Background Violence is the intentional use of physical force or power, threatened or actual, against oneself, another person, a group or community that either results in or has a high likelihood of resulting in injury, psychological harm or death. The objective of the study was to determine the incidents \& risk factors for gender based violence in South Africa.

Methods This study was a cross sectional study. Data was collected by trained volunteers and supervised by appointed supervisors and investigators, by a face-to-face interview using a pre-tested structured questionnaire on GBV. Frequency count was generated for all variables and statistical test of significance was performed with Chi-Square test.

Results A total of 145 consenting respondents participated with a mean age \pm SD of $31.93 \pm 11.26$ years. $73(50.3 \%)$ have experienced physical violence with 47 (32.4\%) beaten, slapped and stabbed \& $29(20.0 \%)$ of the incidents occurring within the last 6 months. 34 (23.4\%) have experienced sexual violence mostly sexual touch (breast/buttock), attempted rape \& rape. $21(14.5 \%)$ have had an unwanted pregnancy with 6 (4.1\%) aborted. $86(59.3 \%)$ have experienced emotional violence either verbal insult or threat. Partner alcohol consumption is associated with experiencing physical violence $\left(\chi^{2}=\right.$ 4.32, $\mathrm{df}=1, \mathrm{P}=0.001$ ) with higher odds (OR: $2.01,95 \%$ CI: 1.04 - 3.89).

Conclusion Gender-based violence is common in South Africa with alcoholism being a serious risk factor for this violence in the society thus alcohol control law implementation is key to halting this trend.

Disclosure No significant relationships. 